\title{
Influence of Atorvastatin on Plasma Atherogenic Biomarkers
}

\author{
Marek Kucera, Stanislav Oravec and Ludovit Gaspar
}

Additional information is available at the end of the chapter

http://dx.doi.org/10.5772/64794

\begin{abstract}
Patients $(n=40)$ with hypercholesterolaemia (29 females), mean age 63 years, without previous lipid lowering treatment, were treated with atorvastatin $40 \mathrm{mg} /$ day for 3 months. Total cholesterol (TC), low-density lipoprotein cholesterol (LDL-C), highdensity lipoprotein cholesterol (HDL-C), triglycerides (TG), LDL-C subfractions (large LDL-C and small dense LDL-C particles), apolipoprotein A1 (apo A1), apolipoprotein B (apo B), apo B/apo A1 ratio, atherogenic index of plasma (AIP), haematological parameters including mean platelet volume (MPV), and red cell distribution width (RDW) and safety parameters (renal and hepatic function) were measured before and after 12 weeks of atorvastatin treatment. Atorvastatin significantly reduced small dense LDL (sdLDL) fraction 3-7 and apo B. There was a negative correlation of AIP with buoyant LDL $1-2(r=-0.35 ; p<0.05)$ and positive with small dense LDL 3-7 $(r=0.52, p$ $<0.001)$. Administration of atorvastatin $40 \mathrm{mg} /$ day in patients with hypercholesterolaemia caused a shift in small dense LDL subfractions to large, buoyant subfractions. AIP correlated better with small dense LDL than apo B levels. At baseline, a strong correlation between HDL-C, TG, small dense LDL-C, apo B, apo B/apo A1 and AIP with MPV was found. After 12 weeks of treatment with atorvastatin, MPV and RDW values underwent significant modification only in those patients displaying the strongest lipidlowering effect. Values of MPV and RDW seem to reflect a pro-atherogenic lipoprotein profile mainly represented by the presence of small dense LDL-C. No serious atorvastatin adverse events were noted.
\end{abstract}

Keywords: atorvastatin, small dense LDL, atherogenic dyslipidaemia, mean platelet volume, red cell distribution width 


\section{Introduction}

Abnormal lipid metabolism preceding overt atherosclerosis is associated with increased cardiovascular risk. In atherogenic dyslipidaemia, the lipoprotein abnormalities include increased small dense LDL particles (sdLDL), elevations of VLDL and low HDL-cholesterol usually occur together [1, 2]. Over the last two decades, it has been demonstrated that routine measurement of total cholesterol, LDL-C and HDL-C fails to distinguish all lipoprotein abnormalities associated with cardiovascular diseases [3]. There is a need to find new biomarkers for this. By contrast, the analysis of lipoprotein subfractions appears more important in assessing the risk of cardiovascular complications [4]. LDL-C remains the primary focus for cardiovascular risk assessment and evaluation of pharmacologic effectiveness, but not based on LDL targets instead on LDL lowering [5]. Yet, a large body of evidence indicates that a narrow focus on LDL-C assessment and treatment alone is not the optimal strategy for patient care [6]. Examining individual lipoprotein subpopulations/subfractions provides opportunities for risk stratification, independent of commonly determined lipid parameters [7].

\section{Plasma atherogenic biomarkers}

The term "lipid triad" has been introduced to describe a common form of dyslipidaemia, characterized by three lipid abnormalities: increased plasma triglyceride levels, decreased HDL-cholesterol concentrations and the presence of sdLDL particles [8]. Apolipoprotein B (apo B) is the major protein of all lipoproteins except for high-density lipoprotein. Estimation of apo B reflects the total number of sdLDL particles. It is notable that LDL particles can vary in size, cholesterol content and number, for a given concentration of LDL-C.

Haematological parameters, mainly red cell distribution width (RDW) and mean platelet volume (MPV) have gained great interest in cardiovascular research. This has been reported to be a strong and independent predictor of adverse cardiovascular outcomes in the general population [9]. In the last few years, MPV, respected an effective marker of platelet activation, has also created much interest in cardiovascular research. This stems from the fact that platelets undergo a dramatic change in shape from quiescent discs to swollen spheres, with an increased MPV, during the activation process. It is well-known that large platelets are more adhesive and prone to aggregate than smaller ones [10], and elevated MPV values have been reported in cardiovascular diseases [11]. "Another haematological parameter which seems to play a role is RDW, a measure of the variability of red cell size. RDW has been reported to be a strong and independent predictor of adverse cardiovascular outcomes in the general population" [12].

The aim of our study was to compare different methods in the evaluation of atherogenicity, including that of the detailed lipid profile. 


\section{Analytic system}

Various methods have been developed such as gradient gel electrophoresis, ultracentrifugation, magnetic resonance spectroscopy, endothelial models for testing lipoprotein cytotoxicity to identify atherogenic lipoproteins [13], but because of technical and financial limitations, long-term analyses and high operating costs, the previously mentioned methods were used primarily in basic research. Electrophoresis of plasma lipoproteins on the polyacrylamide gel (PAG) Lipoprint LDL system is a new method, which has become a milestone in routine laboratory analysis and in diagnosing disorders of lipoproteins, for the identification and quantitative evaluation of lipoprotein subfractions, i.e., the atherogenic and non-atherogenic lipoproteins [14]. The benefits of Lipoprint LDL method are in unique identification of an atherogenic and non-atherogenic lipoprotein spectrum in case of hyperlipoproteinaemia with the possibility of a better assessment of the adequacy of lipid-lowering interventions. Another important aspect is to identify the atherogenic lipoprotein profile in patients with normolipidaemia after lipid-lowering therapy [15].

\section{Cholesterol lowering, atherogenic biomarker alteration and therapy}

Clinical trials with statins have demonstrated significant reductions in cardiovascular events. Although the benefit of statin therapy has generally been ascribed to reduction in LDL-C, other atherogenic classes of lipoproteins may be beneficially affected by statin therapy [16]. The beneficial effects of atorvastatin treatment have been known for a long time although, the effect of atorvastatin on other classes of atherogenic lipoproteins has not been well studied. Previous studies, which were performed in limited trials of patients with atorvastatin 10 and $20 \mathrm{mg}$, resulted in a shift from small to large atherogenic particles of low-density lipoprotein (LDL) $[17,18]$. We know that atorvastatin can improve lipoprotein metabolism, however, the medication affects different aspects of lipoprotein metabolism.

Lipid lowering treatment changes the sizes and concentrations of subtype lipoproteins and the values of some haematological parameters. There are more another favourable effects of statins in regulation of coagulation, inflammation and vascular function instead reducing LDL-C $[19,20]$. Therefore, the influence of atorvastatin in decreasing cardiovascular risk could be through reduction of MPV levels. The antiplatelet and anti-inflammatory effect of atorvastatin could play a role in decreasing cardiovascular risk by reduction of MPV levels [21]. Patients with low HDL-C have significantly higher levels of MPV [22]. Likewise, the negative association has been revealed between increased RDW and low HDL-C values evaluated in large outpatient trials [23]. However, there are little data evaluating the association between MPV, RDW and low-density lipoproteins values.

A further aim of the present survey was to identify the relationship between low-density lipoprotein subfractions and haematologic parameters. The point of interest was to examine whether MPV and RDW have predictive potential for plasma levels and composition of proor anti-atherogenic lipoproteins. A cohort of 40 patients with hypercholesterolaemia (29 
females, mean age $62.9 \pm 9$ years), without previous hypolipidaemic treatment were enrolled. The patients were treated with atorvastatin $40 \mathrm{mg} /$ day for 12 weeks. There was documented hypercholesterolaemia in 21 patients, combined with hyperlipoproteinaemia in 19 of those.

\subsection{Study design}

All participants signed informed content and went through a screening protocol which included evaluation of their medical history, physical examination and testing for standard haematologic and biochemical analysis. Exclusion criteria were a history of diabetes mellitus, glomerular filtration rate less than $60 \mathrm{ml} / \mathrm{min}$ (estimated glomerular filtration rate using MDRD equation $[\mathrm{GFR}]<60 \mathrm{ml} / \mathrm{min}$ ) and liver abnormalities (abnormal AST, ALT, history of hepatopathy or cirrhosis), history of acute myocardial infarction or stroke, hypothyroidism or hyperthyroidism (abnormal TSH), cancer, history of pancreatitis, alcohol or drug abuse, systemic connective tissue diseases, history of anaemia, red blood cell transfusion, supplementation of iron, folate or stimulation of erythropoiesis. Patients were eligible to take part in the study if they met the criteria of the National Cholesterol Education Program-Adult Treatment Panel 3 (NCEP-ATP3) [24]. None of the patients involved in the study were treated with a statin before, even though some already had a diagnosis of hypertension or ischaemic heart disease. Physical and laboratory examinations were carried out after first, second and third month of treatment. The study was approved by the local ethics committee of the University Hospital in Bratislava and was conducted in accordance with the Declaration of Helsinki.

\subsection{Measurements}

Blood samples were drawn from the cubital vein in the morning after $12 \mathrm{~h}$ fasting period. Monitoring included laboratory screening (liver and renal function, glucose, electrolytes, thyroid stimulating hormone), identification of plasma atherogenity-apolipoprotein B, apolipoprotein A1, ratio apo B/apo A1 by immunoturbidimetric method (Roche, Germany) and atherogenic index of plasma (AIP) using the formula log (triglycerides [TG]/high-density lipoprotein cholesterol [HDL-C]).

The lipoprotein subpopulations - VLDL (very low-density lipoprotein), IDL (intermediate density lipoprotein): IDL1, IDL2, IDL3 (the PAG method separates the intermediate-density lipoprotein particles into three midbands MID-C, MID-B and MID-A on figures), LDL 1 to LDL 7 and HDL (high-density lipoprotein) were determined by the linear electrophoresis in polyacrylamide gel (Quantimetrix Lipoprint LDL System and Quantimetrix, California, USA). The type of lipoprotein spectrum was determined as non-atherogenic profile versus atherogenic profile. LDL 1 and LDL 2 were classified as large particles (non-atherogenic) and LDL 3 to 7 as sdLDL particles (atherogenic).

Haematological variables (including MPV, RDW) before and after treatment were measured by cell analysers (Sysmex Haematology Analyzer XP-2000i, Japan). The normal range of MPV (fl) and RDW (\%) in our laboratory was 7.8-11 and 10.0-15.2, respectively. The blood samples 
were collected in tripotassium EDTA tubes and time delay between sampling and data analysis was strictly controlled to be less than $2 \mathrm{~h}$.

\subsection{Statistical analysis}

GraphPad Prism 5 software for Windows was used. The D'Agostino Pearson test and Kolmogorov-Smirnov test were used to verify the normal distribution of parameters in the cohort. Continuous variables were expressed as mean \pm SD or median and interquartile range. We used Spearman's and Pearson's correlation analyses. For the 40 patients who were studied before and after 3 months of atorvastatin treatment, unpaired $t$ tests were performed to compare apo A1, apo B, AIP and serum lipoproteins at baseline versus after 3 months of treatment. The effect of 12 weeks treatment with atorvastatin on lipid and haematological parameters was evaluated by using paired $t$ test and Wilcoxon matched-pairs signed rank test. A two-tailed probability level $<0.05$ was considered significant.

\subsection{Results}

Patients in the study were diagnosed with dyslipoproteinaemia, in which $52.5 \%$ patients $(n=$ 21) was found isolated hypercholesterolaemia and the other patients $(47.5 \%, n=19)$ combined with hyperlipidaemia. Isolated hypertriglyceridaemia was not detected. The baseline characteristics of the participants are presented in Table 1. When structuring groups of patients according to atherogenic and non-atherogenic lipoprotein profiles, hypercholesterolaemia without the presence of sdLDL was found in 29 subjects $(72.5 \%)$, atherogenic sdLDL in 11 persons $(27.5 \%)$, whereas $42 \%$ of patients with combined hyperlipaemia $(n=8)$ and only $14 \%$ of patients with isolated hypercholesterolaemia $(n=3)$ had atherogenic lipoprotein profile phenotype. We also observed 35\% $(n=14)$ of individuals with hyperlipoproteinaemia LDL 1 , 2 with non-atherogenic lipoprotein profile phenotype.

\begin{tabular}{|c|c|c|c|c|}
\hline \multirow{3}{*}{ Number $n(\%)$} & \multicolumn{2}{|l|}{ Men } & \multicolumn{2}{|l|}{ Women } \\
\hline & \multicolumn{2}{|c|}{$11(27.5 \%)$} & \multicolumn{2}{|l|}{$29(72.5 \%)$} \\
\hline & $\begin{array}{l}\text { Min- } \\
\max \end{array}$ & Mean \pm SD & $\begin{array}{l}\text { Min- } \\
\max \end{array}$ & Mean \pm SD \\
\hline Age (years) & $42-72$ & $60 \pm 9.0$ & $47-85$ & $63.6 \pm 9.1$ \\
\hline BMI $\left(\mathrm{kg} / \mathrm{m}^{2}\right)$ & $\begin{array}{l}23.2- \\
33.7\end{array}$ & $28.6 \pm 3.1$ & $20.8-39.1$ & $26.7 \pm 4.7$ \\
\hline Waist (cm) & $\begin{array}{l}78- \\
108\end{array}$ & $94.6 \pm 12.0$ & $\begin{array}{l}74.0- \\
113.0\end{array}$ & $87.2 \pm 10.2$ \\
\hline Smokers, $n(\%)$ & \multicolumn{2}{|c|}{$4(36.4 \%)$} & \multicolumn{2}{|l|}{$4(13.8 \%)$} \\
\hline $\mathrm{CAD}, n(\%)$ & \multicolumn{2}{|c|}{$2(18.2 \%)$} & \multicolumn{2}{|l|}{$4(13.8 \%)$} \\
\hline Arterial hypertension, $n(\%)$ & \multicolumn{2}{|c|}{$8(72.5 \%)$} & \multicolumn{2}{|l|}{$22(75.9 \%)$} \\
\hline Obesity, $n(\%)$ & \multicolumn{2}{|c|}{$3(27.3 \%)$} & \multicolumn{2}{|l|}{$6(20.7 \%)$} \\
\hline
\end{tabular}

BMI, body mass index; CAD, coronary artery disease; SD, standard deviation.

Table 1. Baseline characteristics of patients included in the study. 


\begin{tabular}{lllll}
\hline & AIP & apo B & apo A1 & apo B/apo A1 \\
\hline LDL 1-2 & -0.35 & 0.54 & 0.13 & 0.358 \\
& $p<0.05$ & $p<0.001$ & NS & $p<0.05$ \\
sdLDL 3-7 & 0.52 & 0.64 & -0.23 & 0.62 \\
& $p<0.001$ & $p<0.001$ & NS & $p<0.001$ \\
HDL & -0.75 & -0.43 & 0.58 & -0.60 \\
& $p-0.001$ & $p<0.05$ & $p<0.001$ & $p<0.001$ \\
IDL 1-3 & 0.14 & 0.19 & -0.19 & 0.18 \\
& $\mathrm{NS}$ & $p<0.05$ & $\mathrm{NS}$ & $\mathrm{NS}$ \\
\hline
\end{tabular}

AIP, atherogenic index of plasma; apo, apolipoprotein; LDL, low-density lipoprotein; sdLDL, small dense low-density lipoproteins; HDL, high-density lipoprotein; IDL, intermediate density lipoprotein; NS, not significant.

Table 2. Correlation between subpopulations and AIP, apo B, apo A1 and apo B/apo A1.

\begin{tabular}{llll}
\hline & Before treatment & After treatment & $p$ \\
\hline TC $(\mathrm{mmol} / \mathrm{l})$ & $6.7 \pm 1.0$ & $4.6 \pm 1.3$ & $<0.001$ \\
TG $(\mathrm{mmol} / \mathrm{l})$ & $1.8 \pm 0.9$ & $1.5 \pm 1.0$ & $<0.05$ \\
LDL-C (mmol/l) & $4.3 \pm 1.0$ & $2.6 \pm 0.9$ & $<0.001$ \\
HDL-C (mmol/l) & $1.4 \pm 0.4$ & $1.2 \pm 0.3$ & $<0.001$ \\
apo A1 (g/l) & $1.68 \pm 0.26$ & $1.63 \pm 0.25$ & $\mathrm{NS}$ \\
apo B (g/l) & $1.00 \pm 0.25$ & $0.74 \pm 0.22$ & $<0.001$ \\
apo B/apo A1 & $0.59 \pm 0.20$ & $0.45 \pm 0.16$ & $<0.001$ \\
AIP & $0.03 \pm 0.30$ & $0.03 \pm 0.31$ & $\mathrm{NS}$ \\
Fasting glucose (mmol/l) & $5.69 \pm 1.53$ & $\mathrm{NS}$ \\
hsCRP (mg/l) & $5.69 \pm 1.31$ & $2.24 \pm 2.65$ & $<0.05$ \\
BMI $\left(\mathrm{kg} / \mathrm{m}^{2}\right)$ & $3.20 \pm 3.58$ & $26.9 \pm 4.3$ & $<0.05$ \\
Waist (cm) & $27.2 \pm 4.3$ & $88.7 \pm 11.1$ & $\mathrm{NS}$ \\
SBP (mmHg) & $89.2 \pm 11.1$ & $126.6 \pm 9.4$ & $<0.05$ \\
DBP (mmHg) & $131.2 \pm 12.4$ & $72.9 \pm 9.3$ & $<0.05$ \\
VLDL (mmol/l) & $76.4 \pm 8.8$ & $0.67 \pm 0.32$ & $<0.001$ \\
IDL 1-3 (mmol/l) & $0.95 \pm 0.36$ & $1.16 \pm 0.33$ & $<0.001$ \\
LDL 1-2 (mmol/l) & $1.63 \pm 0.38$ & $1.55 \pm 0.65$ & $<0.001$ \\
sdLDL 3-7 (mmol/l) & $2.50 \pm 0.76$ & $0.09 \pm 0.16$ & $<0.001$ \\
\hline TC & $0.22 \pm 0.32$ & \\
\hline
\end{tabular}

TC, total cholesterol; TG, triglycerides; LDL, low-density lipoprotein; sdLDL, small dense low-density lipoproteins; HDL, high-density lipoprotein; apo A1, apolipoprotein A1; apo B, apolipoprotein B; AIP, atherogenic index of plasma; SBP, systolic blood pressure; DBP, diastolic blood pressure; BMI, body mass index; hsCRP, highly sensitivity C-reactive protein; VLDL, very low-density lipoprotein; IDL, intermediate density lipoprotein.

Table 3. Effect of atorvastatin on lipids, apolipoproteins, fasting glucose, hsCRP, AIP and other parameters. 
We observed a positive correlation between apo B and sdLDL 3-7 $(r=0.64, p<0.001)$, and with LDL $1-2(r=0.54, p<0.001)$. AIP showed a negative correlation with LDL $1-2(r=-0.35, p<$ $0.05)$ and was positively correlated with sdLDL 3-7 $(r=0.52, p<0.001$; Table 2$)$. There was a non-significant change of the atherogenic index of plasma $(0.03 \pm 0.30$ versus $0.03 \pm 0.31, p=$ 0.142), significant decrease in high-sensitivity C-reactive protein (hsCRP) (3.20 \pm 3.58 versus $2.24 \pm 2.65 \mathrm{mg} / \mathrm{l}, p<0.05)$ and body mass index (BMI) $\left(27.2 \pm 4.3\right.$ versus $26.9 \pm 4.3 \mathrm{~kg} / \mathrm{m}^{2}, p<$ 0.05 ; Table 3).

A.

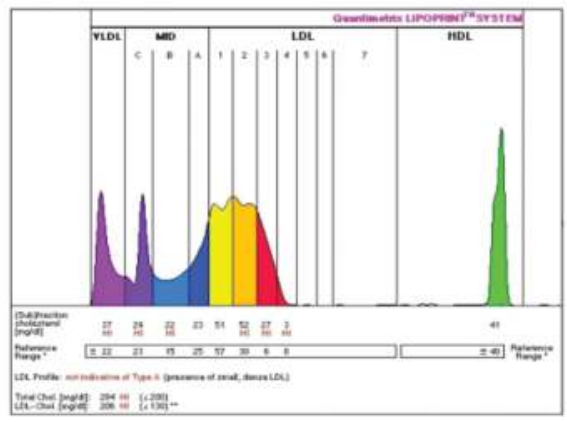

B.

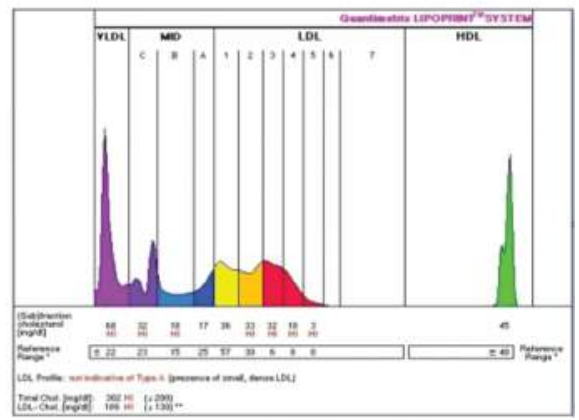

Figure 1. Distribution of lipoproteins using polyacrylamide gel method before (A) and after (B) treatment with atorvastatin $40 \mathrm{mg}$. Atherogenic lipoproteins are present despite treatment (MID-C is IDL-1, MID-B is IDL-2 and MID-A is IDL-3).

A.

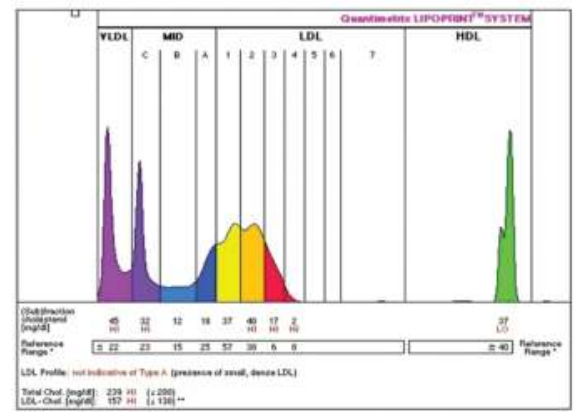

B.

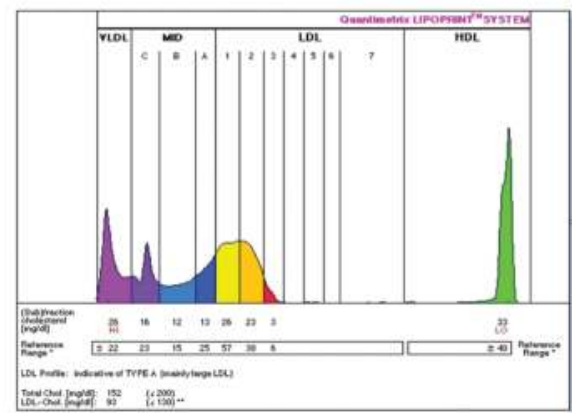

Figure 2. Reduction of lipoprotein subpopulations after treatment (B) with atorvastatin $40 \mathrm{mg}$ compared with baseline lipid lipoprotein profile (A). There is fall in the atherogenic LDL 3-7 fraction after treatment (MID-C is IDL-1, MID-B is IDL-2 and MID-A is IDL-3).

Despite lipid-lowering therapy and normal values of lipids, the Lipoprint LDL method revealed presence of sdLDL (Figure 1). Atorvastatin significantly reduced the presence of sdLDL $3-7(0.22 \pm 0.37$ versus $0.09 \pm 0.16 \mathrm{mmol} / \mathrm{l}, p<0.001)$, as well as other subpopulations of 
lipoproteins (LDL 1-2, VLDL, IDL 1-3; Figure 2). Atorvastatin $40 \mathrm{mg}$ had little effect on the initial subfractions LDL 1 and LDL 2 (Figure 3). The proportion of buoyant and sdLDL at baseline was $11.36 \mathrm{mmol} / \mathrm{l}(p<0.001)$ and after treatment $17.22 \mathrm{mmol} / \mathrm{l}(p<0.001)$.

A.

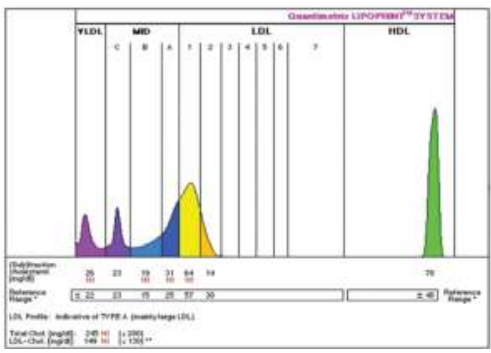

B.

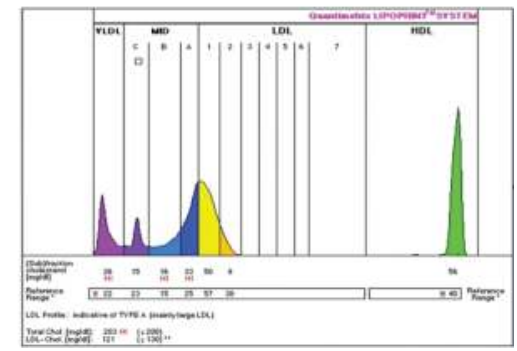

Figure 3. Effect of atorvastatin $40 \mathrm{mg}$ on hyperbetalipoproteinaemia before (A) and after treatment (B). There were no changes in lipoprotein profile after treatment (MID-C is IDL-1, MID-B is IDL-2 and MID-A is IDL-3).

\begin{tabular}{lllc}
\hline Haematological parameters & $\begin{array}{l}\text { Before treatment } \\
\text { Mean } \pm \text { SD/Median [IOR] }\end{array}$ & $\begin{array}{l}\text { After treatment } \\
\text { Mean } \pm \text { SD/Median [IOR] }\end{array}$ & $P$ \\
\hline RBC $\left(10^{12} / 1\right)$ & $4.16[3.9-4.7]$ & $4.12[3.9-4.69]$ & NS \\
Haemoglobin $(\mathrm{g} / \mathrm{l})$ & $143.0[139.0-148.8]$ & $143.0[137.3-147.8]$ & NS \\
MCV (fl) & $90.45[89.03-92.1]$ & $90.0[88.93-91.52]$ & NS \\
RDW $(\%)$ & $12.75[11.0-15.5]$ & $12.4[10.85-15.35]$ & NS \\
Platelet $\left(10^{9} / 1\right)$ & $288.0[217.0-300.5]$ & $288.0[256.5-309.8]$ & $<0.05$ \\
MPV (fl) & $8.65[8.03-10.68]$ & $8.80[8.33-9.63]$ & NS \\
WBC $\left(10^{9} / 1\right)$ & $6.42[5.91-7.4]$ & $6.6[6.0-7.34]$ & NS \\
\hline
\end{tabular}

RBC, red blood cell count; MCV, mean cell volume; RDW, red cell distribution width; MPV, medium platelet volume; WBC, white blood cell count; IQR, interquartile range; $r$, correlation index.

$p<0.05$ was significant; NS, non-significant.

Table 4. Comparison of haematological parameters before and after atorvastatin treatment.

In all subjects, atorvastatin treatment was administered as either primary or secondary prevention. By contrast, no significant changes of the selected haematological parameters were observed after statin intervention, except for platelet count (Table 4). However, when applying the correlation analysis, a strong association between the haematological parameters and plasma lipids was evident at baseline and after 12 weeks of statin therapy (Tables 5 and 6). In particular, in the subgroup of patients $(n=25)$ experiencing the greatest lipid-lowering effect, as determined by the reduction of sdLDL-C, a statistically significant decline of MPV $(p=0.006)$ was observed. A positive association between the levels of sdLDL-C and MPV in this cohort 
was clearly evident at baseline $(r=0.83, p<0.001)$ and after therapy $(r=0.7, p<0.001$; Table 7). In addition, haemoglobin and platelet count appeared modified following a course of statin treatment ( $p=0.003, p=0.03$, respectively) in this subgroup of patient. Atorvastatin was well-tolerated during the study. No serious adverse events were noted.

\begin{tabular}{llllll}
\hline Baseline & MPV & RDW & After treatment & MPV & RDW \\
\hline Total cholesterol & $r=0.04$ & $r=0.10$ & Total cholesterol & $r=-0.03$ & $r=0.05$ \\
& NS & NS & & NS & NS \\
LDL-C & $r=0.07$ & $r=0.08$ & LDL-C & $r=-0.19$ & $r=0.07$ \\
& NS & NS & & NS & NS \\
HDL-C & $r=-0.55$ & $r=-0.49$ & HDL-C & $r=-0.37$ & $r=-0.39$ \\
& $p<0.001$ & $p<0.001$ & & $p<0.05$ & $p<0.05$ \\
TG & $r=0.57$ & $r=0.62$ & TG & $r=0.31$ & $r=0.39$ \\
& $p<0.001$ & $p<0.001$ & & $p<0.05$ & $p<0.05$ \\
\hline
\end{tabular}

LDL-C, low-density lipoprotein; HDL-C, high-density lipoprotein; TG, triglycerides; MPV, medium platelet volume; RDW, red cell distribution width; $r$, correlation index; NS, non-significant.

$p<0.05$ was significant.

Table 5. Correlation between values of plasma lipids and haematological parameters at baseline and after treatment.

\begin{tabular}{llllll}
\hline Baseline & MPV & RDW & After treatment & MPV & RDW \\
\hline LDL 1-2 & $r=-0.05$ & $r=-0.21$ & LDL 1-2 & $r=0.01$ & $r=0.12$ \\
& NS & NS & & NS & NS \\
sdLDL 3-7 & $r=0.73$ & $r=0.67$ & sdLDL 3-7 & $r=0.54$ & $r=0.56$ \\
& $p<0.001$ & $p<0.001$ & & $p<0.001$ & $p<0.001$ \\
IDL 1-3 & $r=-0.05$ & $r=0.04$ & IDL 1-3 & $r=-0.21$ & $r=0.1$ \\
& NS & NS & & NS & NS \\
apo_B & $r=0.41$ & $r=0.41$ & apo B & $r=-0.03$ & $r=0.26$ \\
apo_A1 & $p<0.05$ & $p<0.05$ & & NS & NS \\
apo B/apo A1 & $r=-0.36$ & $r=-0.24$ & apo_A1 & $r=-0.19$ & $r=-0.26$ \\
& $p<0.05$ & NS & & NS & NS \\
AIP & $r=0.52$ & $r=0.43$ & apo B/apo_A1 & $r=0.03$ & $r=0.39$ \\
& $p<0.001$ & $p<0.05$ & & NS & $p<0.05$ \\
\hline
\end{tabular}

LDL, low-density lipoprotein; sdLDL, small dense low-density lipoproteins; IDL, intermedium density lipoprotein; apo_B, apolipoprotein B; apo_A1, apolipoprotein A1; AIP, atherogenic index of plasma; MPV, medium platelet volume; RDW, red cell distribution width; $r$, correlation index; NS, non-significant. $p<0.05$ was significant.

Table 6. Correlation between lipoproteins, apolipoproteins, AIP and haematological parameters at baseline and after treatment. 


\begin{tabular}{|c|c|c|c|}
\hline $\begin{array}{l}\text { Haematological parameters in the subgroup } \\
\text { (n } n==25 \text { ) }\end{array}$ & $\begin{array}{l}\text { Before treatment } \\
\text { Mean } \pm \text { SD/Median } \\
{[\text { IOR] }}\end{array}$ & $\begin{array}{l}\text { After treatment } \\
\text { Mean } \pm \text { SD/Median [IOR] }\end{array}$ & $p$ \\
\hline $\operatorname{RBC}\left(10^{12} / 1\right)$ & $4.46 \pm 0.46$ & $4.44 \pm 0.46$ & NS \\
\hline Haemoglobin (g/l) & $145.8 \pm 5.61$ & $144.9 \pm 5.81$ & $p<0.05$ \\
\hline $\operatorname{MCV}(\mathrm{fl})$ & $90.8 \pm 2.15$ & $90.21 \pm 1.74$ & NS \\
\hline RDW (\%) & $13.30[12.2-15.85]$ & $13.0[11.5-15.8]$ & NS \\
\hline Platelet $\left(10^{9} / 1\right)$ & 287.0 [194.0-298.0] & $287.0[209.0-306.0]$ & $p<0.05$ \\
\hline MPV (fl) & 9.9 [8.4-10.9] & 8.9 [8.45-9.85] & $p<0.05$ \\
\hline WBC $\left(10^{9} / 1\right)$ & 6.34 [5.98-6.95] & 6.6 [5.95-6.8] & NS \\
\hline
\end{tabular}

RBC, red blood cell count; MCV, mean cell volume; RDW, red cell distribution width; MPV, medium platelet volume; WBC, white blood cell count; IQR, interquartile range; $r$, correlation index; NS, non-significant. $p<0.05$ was significant.

Table 7. Comparison of haematological parameters before and after atorvastatin treatment.

\subsection{Discussion}

Over last decade, evidence from clinic trials indicates that broad-based treatment of dyslipidaemia can improve the event-free survival rate in people who already have clinical cardiovascular diseases [25]. However, assessment of atherogenesis requires the quantification small dense lipoproteins both in patients with hyperlipoproteinaemia and normolipaemia. The decrease in HDL-C level we observed requires further study. It also seems necessary to focus on the changes of HDL subpopulations during lipid-lowering therapy [26]. A substantial residual cardiovascular risk persists, despite best treatment efforts [27-29]. We observed a 35\% occurrence of hyperlipoproteinaemia LDL 1 and LDL 2 with non-atherogenic lipoprotein profile phenotype A in our patients. LDL 1 and LDL 2 are less atherogenic or not atherogenic at all and are responsible for the transport of cholesterol [30]. The presence of LDL 1 and LDL 2 in serum in the optimal concentration is essential for the normal function of endocrine organs with steroidogenesis, but also for the formation of bile acids, vitamin D3, enzymes of lipid metabolism and cell renewal of membrane structures. Within cells, cholesterol is important precursor molecule for several biochemical pathways. There is necessary for their physiological processes in the body [31]. It would be desirable for future studies to directly explain this non-atherogenic hyperbetalipoproteinaemia LDL1,2 in hypercholesterolaemic subjects with cardiometabolic diseases.

"Atherogenic normolipidaemia" after statin therapy is crucial, as this profile may increase cardiovascular risk. However, the usefulness of searching for atherogenic lipoproteins in these 
patients requires further research. "Atherogenic normolipaemia" was not present in our subjects, because we chose the patients for atorvastatin therapy according to the recommendations for the treatment of dyslipoproteinaemia [32,33]. However, a breakthrough in the stage of subclinical atherosclerosis may not occur unless we are able to clearly quantify atherogenic lipoproteins both in patients with hyperlipoproteinaemia and normolipaemia.

In our study, a correlation between apo B and lipoproteins was determined. Apo B was raised not only in the presence of sdLDL 3-7 but also in the case of large LDL 1-2 particles. The only protein component of LDL is a single molecule of apo B-100 per particle [34]. Apolipoprotein $B$ therefore cannot provide direct information about the density and size of particles in patients in whom excess of sdLDL particles can be expected [33]. This is not common practice. Therefore, the number of LDL particles could be more important in terms of risk than particle size in itself as a better option for diagnosis of atherogenic particles. If further studies this option confirmed, it seems that the easiest marker of atherogenicity could be the AIP [35]. Our results show a significant change in LDL particle size (from larger and cholesterol-rich to smaller and cholesterol-poor) after atorvastatin treatment. We wanted to point out the persistence of "atherogenic normolipaemia" and hypercholesterolaemia in some patients irrespective of statin therapy. Therefore, qualitative determination of lipoproteins after lipid-lowering therapy was the aim of the study [36].

A lot of evidence support that statins have pleiotropic effects beyond their cholesterol-lowering activity [37, 38]. For instance, statins modulate platelet function via direct interactions with platelet membranes [39] or regulation of platelet signalling pathways [40]. Increased platelets activity is crucial in pathophysiology of atherothrombosis. Recent reports showed that statins involve the inhibition of calcium-dependent phospholipase A2 (cPLA2) [41], the activation of nitric oxide synthase [42], and the accumulation of cAMP [43] in the anti-aggregation. Treatment with atorvastatin or simvastatin also causes down-regulation of the expression of CD36 and LOX-1, the reduction of platelet-associated oxidized LDL level [44] and thromboxane A2 and B2 formation [39], and the inhibition of NADPH oxidase (Nox2) [45].

Although MPV is parameter of platelet size, it is considered as a marker of platelet reactivity [46]. Sivri et al. reported that MPV significantly decreased after statin treatment for the irrespective of cholesterol levels [47]. Recent trial indicates the effect of rosuvastatin on MPV level although the changes were not correlated with the plasma lipids which may reflect significant anti-platelet activation properties [48]. Similarly to previous study, the work with atorvastatin has revealed possible relation of statin treatment and MPV [21]. We found that there were no effect of 12 weeks atorvastatin therapy on MPV and RDW, but in the subgroup of patients $(n=25)$ with the decrease in sdLDL-C after statin treatment, we have observed significant reduction in MPV (Table 7).

The question of how we could decrease MPV has already been raised [49]. It has been revealed that the lipid-lowering effect of statin therapy is not involved in inhibition of thrombus formation in hypercholesterolaemic subjects. Previous finding suggests that other lipidindependent effects of statins may contribute to their anti-aggregatory activity [50]. A limitation of MPV, as a prognostic marker, is uncertain up-to-date as the relationship between platelet size and cardiovascular risk is causative or is only a secondary effect. 
The RDW is a measurement of cell size distribution and is commonly requested test used for variety of purposes, for instance to distinguish aneamias [51]. Recent work has revealed a strong correlation between elevated RDW and the occurrence of fatal and nonfatal cardiovascular events [52]. Complete blood count risk score including RDW was strongly associated with all-cause mortality in the JUPITER study in primary populations initially free from cardiovascular disease [53]. Recently, Lippi et al. [23] reported a significant association of increased RDW and low HDL-C, as well as relation with high TG and high AIP involving 4874 unselected outpatients. An interventional study of 79 patients treated with atorvastatin (10$80 \mathrm{mg}$ ) for 24-week period has not shown significant RDW changes [21]. It remains still unknown which physiological process leads to previous outcomes, although inflammation has been reported as possible cause [54]. Therefore, there might be a new and unpredictable scenario in the clinical usefulness of RDW.

It has been suggested that cardiovascular risk may be more closely related to atherogenic lipoprotein profile mostly represented by presence of sdLDL-C particles [55]. To our knowledge, the present study was the first to analyse MPV and RDW in relation to concentration of lipoprotein subfractions, especially with sdLDL-C. The main finding was that MPV and RDW correlated with sdLDL-C and in subgroup of sdLDL-C lowering effect after treatment, indeed, there was significant decrease in MPV value (Table 6). This may indicate that subjects with more a pronounced lipid-lowering effect after statin treatment may benefit also from effects beyond this well-known action. We have also observed that platelet count and haemoglobin value have changed significantly after statin therapy in this subgroup. However, this finding appears not to be clinically important.

The current study results suggest that MPV and RDW can play an important role not only in hypercholesterolaemia but also interfering with atherogenic small dense lipoproteins. Haematological parameters can be easily analysed at low cost and indices be new biomarkers for atherosclerosis. The potential problems that may concern is that various studies revealed the significance of MPV or RDW about the presence of various diseases and confounding factors (obesity, smoking, arterial hypertension, inflammation diseases, pulmonary embolism, etc.). Its clinical significance, however, remains largely difficult. There is still a need for further prospective, multicentre studies with a large sample size to fully clarify the issue.

\section{Acknowledgements}

This work was supported by a grant from the European Regional Development Fund - Project FNUSA-ICRC (No. CZ.1.05/1.1.00/02.0123). We would like to acknowledge the excellent technical assistance of all participants from 2nd Department of Internal Medicine, Faculty of Medicine, Comenius University, Bratislava, Slovak Republic.

\section{Conflict of interest and funding}

The authors declare no conflict of interest and have not received any funding or benefits from industry or any for profit organization for this work. 


\section{Author details}

Marek Kucera*, Stanislav Oravec and Ludovit Gaspar

*Address all correspondence to: marekucera@gmail.com

2nd Department of Internal Medicine, Comenius University in Bratislava, Slovak Republic

\section{References}

[1] Musunuru K. Atherogenic dyslipidemia: cardiovascular risk and dietary intervention. Lipids. 2010; 45: 907-14.

[2] Gang H, Qing Q, Tuomilehto J, et al. Prevalence of the metabolic syndrome and its relation to all-cause and cardiovascular mortality in nondiabetic European men and women. Arch Intern Med. 2004; 164(10): 1066-76.

[3] Ridker PM, Rifai N, Cook NR, Bradwin G, Buring JE. Non-HDL cholesterol, apolipoproteins A-I and B100, standard lipid measures, lipid ratios, and CRP as risk factors for cardiovascular disease in women. JAMA. 2005; 20: 326-33.

[4] Oravec S, Dukat A, Gavornik P, et al. Atherogenic versus non-atherogenic lipoprotein profiles in healthy individuals. is there a need to change our approach to diagnosing dyslipidemia? Curr Med Chem. 2014; 21(25): 2892-901.

[5] Gulati M, Merz CN. New cholesterol guidelines and primary prevention in women. Trends Cardiovasc Med. 2015; 25(2): 84-94.

[6] Oravec S, Gruber K, Dukat A, et al. The assessment of the atherogenic lipoprotein profile in cardiovascular diseases by lipoprint system analysis. In: Kostner G, Chennamesetty I, editors. Biochemistry, Genetics and Molecular Biology “Lipoproteins - From Bench to Bedside". Croatia: InTech; 2015. p. 87-111.

[7] Oravec S, Gruber K, Dostal E. Hyper-betalipoproteinemia LDL 1,2: a newly identified nonatherogenic hypercholesterolemia in a group of hypercholesterolemic subjects. Neuro Endocrinol Lett. 2011; 32(3): 322-7.

[8] Rizzo M, Berneis K. Lipid triad or atherogenic lipoprotein phenotype: a role in cardiovascular prevention? J Atheroscler Thromb. 2005; 12(5): 237-9.

[9] Gijsberts CM, den Ruijter HM, de Kleijn DP, et al. Hematological parameters improve prediction of mortality and secondary adverse events in coronary angiography patients: a longitudinal cohort study. Medicine (Baltimore). 2015; 94(45):1-10. 
[10] Martin JF, Trowbridge EA, Salmon G, Plumb J. The biological significance of platelet volume: its relationship to bleeding time, thromboxane B2 production and megakaryocyte nuclear DNA concentration. Thromb Res. 1983; 32: 443-60.

[11] Pizzulli L, Yang A, Martin JF, Luderttz B. Changes in platelet size and count in unstable angina compared to stable angina or non-cardiac chest pain. Eur Heart J. 1998; 19: 804.

[12] Perlstein TS, Weuve J, Pfeffer MA, Beckman JA. Red blood cell distribution width and mortality risk in a community-based prospective cohort. Arch Intern Med. 2009; 169: 588-94.

[13] Hirany SV, Othman Y, Kutscher P, et al. Comparison of low-density lipoprotein size by polyacrylamide tube gel electrophoresis and polyacrylamide gradient gel electrophoresis. Am J Clin Pathol. 2003; 119: 439-45.

[14] Oravec S. Identifikácia subpopulácií LDL triedy - Aktuálny prínos v diagnostike porúch metabolizmu lipoproteínov a ochorení kardiovaskulárneho systému (Identification of LDL subfractions-a contribution in the diagnostics of lipoprotein metabolism diseases and cardiovascular diseases). Med Milit Slov. 2006; 8: 32-4.

[15] Hoefner MD, Hodel DS, O'Brien FJ, et al. Development of a rapid quantitative method for LDL subfractionation with use of the Quantimetrix Lipoprint LDL system. Clin Chem. 2001; 47(2): 266-74.

[16] Maron DJ, Fazio S, Linton MF. Current perspectives on statins. Circulation. 2000; 101: 207-13.

[17] Sasaki S, Kuwahara N, Kunitomo K, et al. Effects of atorvastatin on oxidized low density lipoprotein, low density lipoprotein subfraction distribution, and remnant lipoprotein in patients with mixed hyperlipoproteinemia. Am J Cardiol. 2002; 89(4): 386-9.

[18] Pontrelli L, Parris W, Adeli K, et al. Atorvastatin treatment beneficially alters the lipoprotein profile and increases low density lipoprotein particle diameter in patients with combined dyslipidemia and impaired fasting glucose in type 2 diabetes. Metabolism. 2002; 51(3): 334-42.

[19] Bickel C, Rupprecht HJ, Blankenberg S, Spinola-Klein C, Rippin G, Hafner G, Lotz J, Prellwitz W, Meyer J, AtheroGene Group. Influence of HMGCoA reductase inhibitors on markers of coagulation, systemic inflammation and soluble cell adhesion. Int J Cardol. 2002; 82: 25-31.

[20] Undas A, Brummel-Ziedins KE, Potaczek DP, Stobierska-Dzierzek B, Bryniarski L, Szczeklik A, Mann KG. Atorvastatin and quinapril inhibit blood coagulation in patients with coronary artery disease following 28 days of therapy. J Thromb Haemost. 2006; 11: 2397-404. 
[21] Akin F, Ayca B, Köse N et al. Effect of atorvastatin on haematologic parameters in patients with hypercholesterolemia. Angiology. 2013; 64: 621-5.

[22] Varol E, Aksoy F, Bas HA, et al. Mean platelet volume is elevated in patients with low high-density lipoprotein cholesterol. Angiology. 2014; 65(8): 733-6.

[23] Lippi G, Sanchis-Gomar F, Danese E, Montagnana M. Association of red blood cell distribution width with plasma lipids in a general population of unselected outpatients. Kardiol Pol. 2013; 71: 931-6.

[24] American College of Cardiology/American Heart Association Task Force on Practice Guidelines. 2013 ACC/AHA guideline on the treatment of blood cholesterol to reduce atherosclerotic cardiovascular risk in adults. Am Coll Cardiol. 2014; 63: 2889-934.

[25] Banach M, Nikfar S, Rahimi R, et al.; Lipid and Blood Pressure Meta-Analysis Collaboration Group. The effects of statins on blood pressure in normotensive or hypertensive subjects - a meta-analysis of randomized controlled trials. Int J Cardiol. 2013; 168(3): 2816-24.

[26] Otocka-Kmiecik A, Mikhailidis DP, Nicholls SJ, Davidson M, Rysz J, Banach M. Dysfunctional HDL: a novel important diagnostic and therapeutic target in cardiovascular disease? Prog Lipid Res. 2012; 51(4): 314-24.

[27] Rizzo M, Barylski M, Rizvi AA, Montalto G, Mikhailidis DP, Banach M. Combined dyslipidemia: should the focus be LDL cholesterol or atherogenic dyslipidemia? Curr Pharm Des. 2013; 19(21): 3858-68.

[28] Rizzo M, Banach M, Montalto G, Mikhailidis DP. Lipid-lowering therapies and achievement of LDL-cholesterol targets. Arch Med Sci. 2012; 8(4): 598-600.

[29] Miller M, Cannon CP, Murphy SA, et al. Impact of triglyceride levels beyond low density lipoprotein cholesterol after acute coronary syndrome in the PROVE-IT TIMI 22 Trial. J Am Coll Cardiol. 2008; 51(7): 724-30.

[30] Oravec S. Plasma lipoproteins. In: Oravec S, editor. Lipoproteins in the diagnosis of internal diseases. Bratislava: VEDA; 1999. p. 31.

[31] Petrov AM, Zefirov AL. Cholesterol and lipid rafts in the biological membranes. Role in the release, reception and ion channel functions. Usp Fiziol Nauk. 2013; 44(1): 17-38.

[32] Banach M, Nikfar S, Rahimi R, et al.; Lipid and Blood Pressure Meta-Analysis Collaboration Group. The effects of statins on blood pressure in normotensive or hypertensive subjects - a meta-analysis of randomized controlled trials. Int J Cardiol. 2013; 168(3): 2816-24.

[33] Walldius G, Jungner I, Holme I, et al. High apolipoprotein B, low apolipoprotein A-1, and improvement in the prediction of fatal myocardial infarction (AMORIS study): a prospective study. Lancet. 2001; 15(9298): 2026-33. 
[34] Mikhailidis DP, Elisaf M, Rizzo M, et al. "European panel on low density lipoprotein (LDL) subclasses": a statement on the pathophysiology, atherogenicity and clinical significance of LDL subclasses. Curr Vasc Pharmacol. 2011; 9(5): 533-71.

[35] Dobiášová M. Atherogenic index of plasma [log(triglycerides/HDL-cholesterol)]: theoretical and practical implications. Clin Chem. 2004; 50(7): 1113-5.

[36] Nikolic D, Katsiki N, Montalto G, Isenovic ER, Mikhailidis DP, Rizzo M. Lipoprotein subfractions in metabolic syndrome and obesity: clinical significance and therapeutic approaches. Nutrients. 2013; 5(3): 928-48.

[37] Liao JK. Effects of statins on 3-hydroxy-3-methylglutaryl coenzyme a reductase inhibition beyond low-density lipoprotein cholesterol. Am J Cardiol. 2005; 96: 24F-33F.

[38] Marzilli M. Pleiotropic effects of statins: evidence for benefits beyond LDL-cholesterol lowering. Am J Cardiovasc Drugs. 2010; 10(1): 3-9.

[39] Ma LP, Nie DN, Hsu SX, et al. Inhibition of platelet aggregation and expression of alpha granule membrane protein 140 and thromboxane B2 with pravastatin therapy for hypercholesterolemia. J Assoc Acad Minor Phys. 2002; 13(1): 23-6.

[40] Luzak B, Rywaniak J, Stanczyk L, Watala C. Pravastatin and simvastatin improves acetylsalicylic acid-mediated in vitro blood platelet inhibition. Eur J Clin Invest. 2012; 42(8): 864-72.

[41] Moscardó A, Vallés J, Latorre A, et al. Reduction of platelet cytosolic phospholipase A 2 activity by atorvastatin and simvastatin: biochemical regulatory mechanisms. Thromb Res. 2013; 131: e154-e159.

[42] Chou TC, Lin YF, Wu WC, Chu KM. Enhanced nitric oxide and cyclic GMP formation plays a role in the anti-platelet activity of simvastatin. Br J Pharmacol. 2008; 153: 12817.

[43] Lee YM, Chen WF, Chou DS, et al. Cyclic nucleotides and mitogen-activated protein kinases: regulation of simvastatin in platelet activation. J Biomed Sci. 2010; 17: 45.

[44] Bruni F, Pasqui AL, Pastorelli M, et al. Different effect of statins on platelet oxidizedLDL receptor (CD36 and LOX-1) expression in hypercholesterolemic subjects. Clin Appl Thromb Hemost. 2005; 11: 417-28.

[45] Pignatelli P, Carnevale R, Pastori D, et al. Immediate antioxidant and antiplatelet effect of atorvastatin via inhibition of Nox2. Circulation. 2012; 126(1): 92-103.

[46] Davi G, Patrono C. Platelet activation and atherothrombosis. N Engl J Med. 2007; 357: 2482-94.

[47] Sivri N, Tekin G, Yalta K, et al. Statins decrease mean platelet volume irrespective of cholesterol lowering effect. Kardiol Pol. 2013; 71: 1042-7. 
[48] Coban E, Afacan B. The effect of rosuvastatin treatment on the mean platelet volume in patients with uncontrolled primary dyslipidemia with hypolipidemic diet treatment. Platelets. 2008; 19(2): 111-4.

[49] Yetkin E. Mean platelet volume not so far from being a routine diagnostic and prognostic measurement. Thromb Haemost. 2008; 100: 3-4.

[50] Gaddam V, Li DY, Mehta JL. Anti-thrombotic effects of atorvastatin-an effect unrelated to lipid lowering. J Cardiovasc Pharmacol Ther. 2002; 7: 247-53.

[51] Evans TC, Jehle D. The red blood cell distribution width. J Emerg Med. 1991; 9: 71-4.

[52] Anderson JL, Ronnow BS, Horne BD, et al. Usefulness of a complete blood countderived risk score to predict incident mortality in patients with suspected cardiovascular disease. Am J Cardiol. 2007; 99: 169-74.

[53] Horne D, Anderson JL, Muhlstein JB, et al. JUPITER trial complete blood count risk score and its components, including RDW, are associated with mortality in the JUPITER trial. Eur J Prevent Cardiol. 2014; 22(4): 519-26.

[54] Tonelli M, Sacks F, Arnold M, et al. Relation between red blood cell distribution width and cardiovascular event rate in people with coronary disease. Circulation. 2008; 117(2): $163-8$.

[55] Arai H, Kokubo Y, Watanabe M, et al. Small dense low-density lipoproteins cholesterol can predict incident cardiovascular disease in an urban Japanese cohort: the Suita study. J Atheroscler Thromb. 2013; 20: 195-203. 
